\title{
O IMPACTO DA ATIVIDADE FÍSICA NA QUALIDADE DE VIDA DO IDOSO
}

\author{
Prof. Dr. Gabriel César Dias Lopes, PhD
}

\section{RESUMO}

A atividade física é importante promotora da qualidade de vida do idoso. No entanto, o tipo de exercício a ser realizado depende do organismo e da vontade de cada um. Atualmente a preocupação com a saúde do idoso tem sido a pauta de instituições e governos preocupados com essa significativa parcela da população. Esse estudo analisa o nível de atividade física que o idoso frequentador do Parque Nacional Serra dos Órgãos em Teresópolis, Rio de Janeiro, desempenha e sua efetiva repercussão em sua saúde. O objetivo é além de traçar esse perfil, diferenciar como homens e mulheres frequentadores do local, realizam as atividades. Trata-se aqui de uma pesquisa bibliográfica baseada em autores que tratam dos temas saúde, bem-estar e atividade física para idosos, usando o método quantitativo para a coleta dos dados ,onde um questionário aplicado a vinte pessoas avaliou o nível dessa atividade física, ficando evidenciado que é satisfatório o índice e que o idoso na cidade começa a buscar os meios para aprimorar sua qualidade de vida, buscando uma velhice agradável, feliz, saudável e ativa.

Palavras-chave: Idoso, Atividade física, Saúde, Qualidade de Vida.

\section{ABSTRACT}

The physical activity is important promoter of the quality of life of the aged one. However, the type of to be carried through exercise depends on the organism and the will on each one. Currently the concern with the health of the aged one has been the guideline of institutions and governments worried about this significant parcel of the population. This study it analyzes the level of physical activity that the aged frequent National Park Mountain range of the Bones in Teresópolis, Rio De Janeiro, it plays and its effective repercussion in its health. The objective is beyond tracing this profile, to differentiate as men and women frequentadores of the place, carry through the activities. One is here about a based bibliographical research in authors who deal with the subjects health, well-being and physical activity for aged, using the quantitative method for the collection of the data, where an applied questionnaire the twenty people evaluated the level of this physical activity, being evidenced that the index is satisfactory and that the aged one in the city starts to search the half ones to improve its quality of life, searching pleasant, happy, healthful and active an oldness.

Word-key: Aged, physical Activity, Health, Quality of Life. 
* Gabriel César Dias Lopes é Ph.D é Graduado em: Teologia, Direito, Administração e Recursos Humanos, MBA em Marketing e Gestão Estratégica, Pós-Graduado em Psicanálise, Pós Graduado em Acupuntura, Pós Graduado em Homeopatia, Coordenador do Curso de Pós Graduação Lato Sensu em Psicanálise Clinica da FABIC, Mestre em Educação, Mestre em Administração, Doutor em Educação e um Ph.D em Psicanálise. Membro da International Special Court of Arbitration and Human Rights - Registro: ISCAHRGCDL-17/11n2016, Membro da Comissão Científica da Olympus Intellectual Center, Atenas (Grecia), Presidente da LUI - Logos University International. 


\section{INTRODUÇÃO}

Estudos demostram que a população idosa no Brasil são hoje 14,5 milhões de pessoas, 8,6\% da população total do País, segundo o Instituto Brasileiro de Geografia e Estatística (IBGE), com base no Censo 2000. O instituto considera idosas as pessoas com 60 anos ou mais, mesmo limite de idade considerado pela Organização Mundial da Saúde (OMS) para os países em desenvolvimento. Em uma década, o número de idosos no Brasil cresceu 17\%, em 1991, ele correspondia a 7,3\% da população.

O envelhecimento da população brasileira é reflexo do aumento da expectativa de vida, devido ao avanço no campo da saúde e à redução da taxa de natalidade. Prova disso é a participação dos idosos com 75 anos ou mais no total da população - em 1991, eles eram 2,4 milhões $(1,6 \%)$ e, em 2000, 3,6 milhões $(2,1 \%)$.

A população brasileira vive, hoje, em média, de 68,6 anos, 2,5 anos a mais do que no início da década de 90. Estima-se que em 2020 a população com mais de 60 anos no País deva chegar a 30 milhões de pessoas (13\% do total), e a esperança de vida, a 70,3 anos. (Prates,2009).

Com o envelhecimento da sociedade, o brasileiro vai conviver mais com idosos, permitindo às gerações que amadurecerem ter um paradigma do que é ser velho. Para combater a inércia à qual os idosos estão confinados, nada melhor do que o movimento. "É uma forma de adiar o repouso absoluto". (POZENA, 2009).

Manter o indivíduo focado nas atividades físicas estabelecendo uma meta de acordo com a idade, peso, altura, adequando-as ao seu genótipo, caminhada, alongamento, exercício resistido sempre acompanhado por um profissional de Educação Física, evitando assim lesões que possam ser adquiridas por uma atividade mal executada tem sido objeto de preocupação de muitos profissionais da área de saúde.

Entende-se que a atividade física ajuda a prevenir agravos em doenças associadas ao sedentarismo, sendo assim o intuito de desenvolver as atividades e promover a educação dos indivíduos, sobre a importância de hábitos saudáveis ambas com pré-requisitos para a saúde geral do ser humano (POZENA, 2009).

A prática regular de exercícios físicos é uma estratégia preventiva primária, atrativa e eficaz, para manter e melhorar o estado de saúde física e psíquica em qualquer idade, tendo efeitos benéficos diretos e indiretos para prevenir e retardar as perdas funcionais do envelhecimento, reduzindo o risco de enfermidades e transtornos freqüentes na terceira idade, 
tais como: as coronariopatias, a hipertensão, a diabetes, a osteoporose, a desnutrição, a ansiedade, a depressão e a insônia.( POZENA, 2009).

Em 1970, a população idosa brasileira era de 5,1\%; dez anos depois, em 1980, já chegava a 6,1\% e em 1990 foi para 7,2\%. Estima-se que em 2005, esta constituia $15 \%$ da população e ainda, que em 2025 será a sexta maior população idosa do mundo, com cerca de 32 milhões de pessoas acima de 60 anos (SHOURI JR. et. al., 1994). Este crescente envelhecimento populacional vem despertando interesse de diversas áreas de estudo.

Partindo dessas premissas questiona-se aqui: qual o nível de atividade física desenvolvido pelos indivíduos com idade a partir de 60 anos que praticam caminhadas no Parque Nacional de Teresópolis? Tendo como principal objetivo, analisar o nível de atividade física dos idosos que praticam caminhada no local supracitado e em segundo plano verificar o nível de atividade física por idade e gênero. Sendo assim, analisar o nível de atividade física de cada indivíduo, através dos procedimentos realizados, e discutir os benefícios relatados após a prática das atividades físicas e as possíveis especificidades encontradas em homens e mulheres, são os objetivos dessa pesquisa.

Nosso intuito é mostrar que a importância dos idosos para o País não se resume à sua crescente participação no total da população. Boa parte dos idosos hoje são chefes de família e nessas famílias a renda média é superior àquelas chefiadas por adultos não-idosos. Segundo o Censo 2000, 62,4\% dos idosos e 37,6\% das idosas são chefes de família, somando 8,9 milhões de pessoas. Além disso, 54,5\% dos idosos chefes de família vivem com os seus filhos e os sustentam.

Uma vez que as doenças nessa faixa etária oneram os cofres públicos nos setores de saúde e previdência social principalmente, é necessário que os governos federal, estadual e municipal estejam atentos para programas de prevenção voltados para essa parcela da população e que eles mesmos, estejam atentos à sua saúde e qualidade de vida.

\section{O ENVELHECIMENTO}

O envelhecimento é um processo dinâmico e progressivo, no qual há alterações morfológicas, funcionais e bioquímicas, com redução na capacidade de adaptação homeostática às situações de sobrecarga funcional, alterando progressivamente o organismo. $\mathrm{O}$ processo de envelhecimento provoca uma série de alterações biológicas, psicológicas e sociais que aumentam a susceptibilidade às doenças e provocam incapacidades. Estudos demonstram que com frequência os idosos são portadores de múltiplas doenças, principalmente as crônico- 
degenerativas, que podem provocar limitações e dependência para Duarte (2003 apud BRITO; LUCIA, 2008, v.17, n.2).

Relata que alterações demográficas são acompanhadas por mudanças no perfil epidemiológico, em que se observa uma redução da incidência de doenças infectocontagiosas e o aumento das doenças crônico-degenerativas, próprias das faixas etárias mais avançadas. Tais transformações exigem novas políticas e novas formas de intervenção no campo da atenção à saúde (2003 apud BRITO; LUCIA, 2008, v.17, n.2).

O grau de independência depende de condições motoras e cognitivas satisfatórias para o desempenho das atividades. O declínio fisiológico da função motora em idosos constitui um dos fatores mais significativos da dependência funcional (GOMES et al, 2004). De acordo com (FERNANDES 1999) a redução da capacidade em executar as atividades básicas da vida diária pode ter consequências para a sobrevivência do idoso, para sua autoestima e sociabilidade.

Devido à acomodação, característica da velhice estereotipada, os idosos passam por problemas que vão aumentando em número e grau com o passar do tempo, entre a diminuição da força muscular e o comprometimento da locomoção, diminui-se também a coordenação motora, tornando as tarefas do cotidiano ainda mais difíceis de serem realizadas. (ALEXANDRE, NICKEL, BORESKIE, E SEARLE, 2000).

Entretanto, para Bolkovoy e Blair (apud SILVA, 1989) esse processo pode ser amenizado com um programa dirigido de atividade física, o que permite aos indivíduos manterem níveis ótimos das funções para sua idade. $\mathrm{O}$ aumento da proporção de idosos na população traz à tona a discussão a respeito de eventos incapacitantes nessa faixa etária (após 60 anos de idade). Esses eventos estão relacionados com a diminuição da capacidade funcional, por exemplo, para a execução das atividades de vida diária, destacando-se a ocorrência de quedas, bastante comum e temida pela maioria das pessoas idosas por suas consequências (BARBOSA, 2001).

Os benefícios provocados pela prática de exercícios físicos pelas pessoas idosas têm sido estudados pela comunidade científica (MATSUDO, 2001), destacando aqueles que atuam na melhora da capacidade funcional, equilíbrio, força, coordenação e velocidade de movimento, contribuindo para uma maior segurança e prevenção de quedas entre as pessoas idosas. $\mathrm{O}$ incremento e/ou a manutenção da atividade física regular podem contribuir para aumentar a expectativa de vida de mulheres idosas, mas parece ter menor benefício nas mulheres menores de 75 anos de idade (ROSENBERG; MOORE,1998).

Uma vez que grande parte dessas evidências epidemiológicas sustenta o efeito positivo de um estilo de vida ativo (e/ou do envolvimento dos indivíduos em programas de atividade 
física ou exercício) na prevenção e minimização dos efeitos deletérios do envelhecimento, os cientistas enfatizam cada vez mais a necessidade da atividade física como parte fundamental dos programas mundiais de promoção da saúde. Não se pode pensar hoje em dia em "prevenir" ou minimizar os efeitos do envelhecimento sem que, além das medidas gerais de saúde, não se inclua também a atividade física (PATHERSON,2002).

Essa preocupação tem sido discutida não somente nos chamados países desenvolvidos ou do primeiro mundo, como também nos países em desenvolvimento, como é o caso do Brasil. Com o avanço da idade ocorre diminuição das atividades cotidianas e redução da funcionalidade, em que a aptidão cardiorrespiratória pode ser considerada um dos componentes mais afetados. O sistema cardiorrespiratório é considerado como um dos sistemas orgânicos mais afetados pelo declínio funcional (BARBOSA et al 2002).

Com o avanço da idade, a habilidade de captação e transporte de oxigênio para o suprimento da demanda metabólica corporal durante a atividade física sustentada torna-se diminuída, influenciando negativamente a saúde e qualidade de vida de idosos. Consequentemente, manter um adequado nível de aptidão cardiorrespiratória (ACR) é indispensável para a manutenção da independência, atenuação da fragilidade e prevenção da dependência (RIKLI RE et al, 1998). Além disso, um nível satisfatório de ACR apresenta um impacto relevante na diminuição do risco para morbidade e/ou mortalidade por todas as causas e por doenças coronarianas (PAFFENBARGER RS, 1986).

O declínio verificado na ACR é influenciado por múltiplos fatores, incluindo o nível de atividade física (MATSUDO, 2001). Uma taxa estimada de declínio relativo na ACR de 10\% por década foi verificada tanto em indivíduos com baixo nível de atividade física (NAF) como em indivíduos com alto NAF.(SPIRDUSO, 2005) Consequentemente, a quantidade de tempo gasta em atividades físicas mais vigorosas diminui com o avanço da idade, podendo reduzir a performance de mobilidade, e uma transição rumo a atividades menos intensas parece ocorrer naturalmente, explicando parcialmente o declínio na ACR(GUIMARÃES, et al, 2006).

Portanto, a habilidade para manter elevado NAF parece ser um fator crítico na manutenção de adequada ACR em sujeitos idosos (MATSUDO SMM, 2001). Stella et al. (2002), enfatizam que a prática de atividade física em grupo eleva a autoestima do idoso, contribui para a implementação das relações psicossociais e para o reequilíbrio emocional. A redução da pressão arterial e da morbimortalidade cardiovascular e vários são os mecanismos envolvidos no efeito hipotensor do treinamento físico, que se torna mais evidente a partir da décima semana de treinamento com pequenos ganhos adicionais subsequentes. (PESCATELLO et al, 2004). 
As vantagens da prática de exercícios para idosos dependem de como se processa o envelhecimento e da rotina de exercício físico praticada. Sabe-se que os benefícios à saúde ocorrem mesmo quando a prática de atividade física é iniciada em uma fase tardia de vida, por sujeitos sedentários, sendo benéfica inclusive para portadores de doenças crônicas prevenindo principalmente as doenças associadas ao sedentarismo, como coronariopatias, diabetes, hipertensão arterial, hipercolesterolêmica, acidente vascular cerebral, osteoporose, osteoartrite, e câncer de próstata, mama e cólon intestinal.

Do efeito dos exercícios na prevenção e recuperação das perdas motoras decorrentes do processo de envelhecimento, conhece-se bem a relação entre treinos específicos e a melhora do órgão ou sistema exercitado, como por exemplo, prática de alongamento muscular e ganho de flexibilidade, ou treino de equilíbrio e melhora no desempenho em testes de equilíbrio. Para que os efeitos do treinamento de exercícios permaneçam, praticá-los deve tornar-se parte da rotina diária.

Os principais motivos relacionados com o início da prática de atividade física regular, independentemente da idade, são o controle ou perda de peso, redução de risco de ocorrência ou recorrência de doenças, principalmente as cardiovasculares, diminuição do estresse ou depressão, melhora da autoestima e socialização. (WEINBERG; GOULD, 1996).

\subsection{Atividade física e o idoso}

Atividade Física é um termo global, definido como todo movimento produzido pela contração da musculatura esquelética e que aumenta, de forma expressiva, o gasto energético. Nesse contexto, a atividade física pode ocorrer no trabalho, em casa durante períodos de lazer e recreação e no trajeto de um lugar para outro (CASPERSEN, 1989). Inclui assim, todas as formas de movimento realizadas no local de trabalho ou de exercício, em casa ou em entidades de assistência a família, no transporte e em espaços de lazer.

Exercício é o movimento corporal planejado, estruturado e repetitivo, feito para melhorar ou manter um ou mais dos componentes do condicionamento físico (CASPERSEN, 1989). Condicionamento físico é um conceito multidimensional, associado a um conjunto de atributos que os indivíduos têm ou alcançam, relacionados com a habilidade de executar as atividades físicas.

Dentre os tipos de condicionamento físico, estão o cardiorrespiratório, o muscular, o metabólico, o motor e o de flexibilidade. Lazer por sua vez, é um conceito que inclui os elementos da livre escolha, ausência de restrições, motivações intrínseca, diversão, 
relaxamento, envolvimento pessoal e oportunidade de auto expressão (HENDERSON, BEALESCHKI, SHAW E FEYSINGER, 1996). Atividade Física volitiva refere-se à atividade feitas com algum propósito, em instalações especialmente preparadas ou não. Em estudos epidemiológicos, em que se mede a atividade física, a frequência, a duração e a intensidade das atividades físicas executadas em determinado período, são estudadas nos termos do seu impacto sobre a saúde. (HENDERSON, BEALESCHKI, SHAW E FEYSINGER, 1996)

Os principais motivos relacionados com o início da prática de atividade física regular, independentemente da idade, são o controle ou perda de peso, redução de risco de ocorrência ou recorrência de doenças, principalmente as cardiovasculares, diminuição do estresse ou depressão, melhora da autoestima e socialização (WEINBERG; GOULD, 1996).

Como principais razões para que sujeitos sedentários há anos, repentinamente, aos 70 ou 80 anos de idade, iniciem e mantenham a prática de exercícios físicos, Shephard (1990), enumera melhora da saúde, aumento da oportunidade de contatos sociais e ganhos na função cerebral. $\mathrm{O}$ autor acredita que o objetivo de melhorar a saúde seja o mais comum e que estaria relacionado com a perda de peso, diminuição do risco de doença cardiovascular e aumento na capacidade da função motora, além dos benefícios nutricionais e melhoria do padrão de sono.

Segundo o autor, muitas pessoas referem exercitar-se porque "isso faz com que se sintam melhor", o que se relaciona com as melhoras de saúde, o aumento na produção de betaendorfinas e aminas biogênicas que atuam no sistema nervoso, reduzindo a ansiedade e produzindo sensação de bem-estar; e as associa, ainda, com modificações positivas da autoimagem, melhora na autoestima e forte sentimento de auto eficácia, que podem induzir a prática de outras atividades de promoção de saúde.

Com a manutenção da atividade física, ocorre a diminuição nos níveis de ansiedade e depressão, influenciando na sensação de bem-estar, principalmente em pessoas com história pregressa dessa sintomatologia.

A frequência está relacionada ao número de vezes semanal, mensal ou anual de atividade física. A duração está relacionada com o tempo gasto em uma atividade especifica como horas ou minutos por sessão. No MRFIT (do inglês Mutipli Risk Factor Inventuration Trial-teste de invenção de Fator de Risco Mútiplo) de 12.138 homens com alto risco de doença cardiovascular, foram executadas análises para determinar a relação entre a atividade física e a mortalidade (LEON, 1987). Mediu-se a atividade física em minutos por dia, classificando-se em três períodos descritos como de curta duração (15 min/dia), média duração (47 min/dia) e longa duração (133 min/dia). Os resultados mostraram menores taxas de mortalidade entre homens que executaram, no tempo de lazer, atividade de média e longa duração, em 
comparação com o grupo de atividade de curta duração. Com base nesse e em outros estudos epidemiológicos revistos por (PATE et al, 1995), são recomendados pelo menos 30 minutos por dia de atividade física para reduzir os riscos de mortalidade e morbidade prematura.

A intensidade refere-se a dificuldade da atividade e, em geral, em questões epidemiológicas, é classificada como leve, moderada ou vigorosa. Ela pode ser expressa em termos absolutos ou relativos. A unidade de intensidade recomendada em termos absolutos é o equivalente metabólico (MET), definido como a razão entre a taxa metabólica da atividade e a taxa metabólica de repouso.

A expressão da intensidade da atividade física em termos relativos cuida do ajuste da dificuldade da atividade de acordo com diferenças de cada indivíduo. As medidas da intensidade relativa incluem o percentual de absorção máxima de oxigênio (\% do Vo2 msx) ou da frequência cardíaca máxima e a classificação do esforço percebido.

A atividade física pode ser medida por uma série de métodos que vão desde medir de forma direta, a quantidade de calor produzida por um corpo durante a atividade, até pedir ao indivíduo para que classifique o grau de atividade em que esteve envolvido na última semana, mês ou ano. (ALVES, 2002)

A prática de atividade física pode controlar e até mesmo evitar alguns sintomas de doenças, ela é um ponto muito importante na vida do idoso, pois segundo Alves (2002) a prática de atividade física, além de combater o sedentarismo, contribui para a manutenção da aptidão física do idoso. A atividade vai influenciar na autonomia do idoso, ele vai começar a realizar atividades que a muito tempo não realizava, podendo se tornar uma pessoa independente.

O outro fator fundamental é a avaliação da saúde do idoso. Estudos mostram que pelo menos $70 \%$ dos idosos têm um problema de saúde e a atividade física pode ser uma grande aliada do tratamento. A prática da atividade física pode controlar a manifestação e os sintomas de várias doenças, como a hipertensão, por exemplo, e reduzir o consumo de remédios. Para isso, é preciso trabalhar com três sistemas do corpo humano: o cardiovascular, o nervoso e o musculoesquelético.

Após essa avaliação o idoso deve escolher a atividade de que mais gosta. É importantíssimo que ele tenha prazer. Ao fazer algo que não gosta, o idoso para após dois meses do início dos exercícios, por isso vale experimentar várias atividades físicas até encontrar a que melhor se adapta ao seu perfil. Entre as atividades que trabalham o sistema cardiovascular estão andar de bicicleta, caminhar e fazer natação. Essas, privilegiam o sistema musculoesquelético a musculação e a ginástica, por exemplo, e atividades como yoga e tai chi chuan, com seus movimentos mais suaves, dão mais atenção ao sistema nervoso. 
A maioria dos idosos prefere fazer caminhadas por dois motivos: não é preciso ter habilidades específicas e é uma atividade que não tem custo. É bom lembrar, que a caminhada não supre todas as necessidades do corpo, pois trabalha apenas o sistema cardiovascular e o musculoesquelético. Esse último fica restrito aos pés e às pernas (SOARES et al., 2006). Por isso é importante ampliar quando possível as possibilidades de atividades além caminhadas.

Ainda de acordo com (SOARES et al., 2006) a redução da capacidade em executar as atividades básicas da vida diária pode ter consequências para a sobrevivência do idoso, para sua autoestima e sociabilidade. É nítido assim, os benefícios da atividade física advindas da mudança de perspectiva e tomada de atitude em prol da saúde por parte do idoso.

Segundo Okuma, (2009) os principais pontos visíveis na melhoria das condições dos idosos são: Melhora da velocidade ao andar e do equilíbrio; Melhora da auto eficácia; Contribuição para a manutenção e/ou o aumento da densidade óssea; Auxílio no controle do diabetes, da artrite, das doenças cardíacas e dos problemas com colesterol alto e hipertensão; Melhora da ingestão alimentar; Diminuição da depressão; Redução da ocorrência de acidentes, pois os reflexos e a velocidade ao andar ficam melhores; Manutenção do peso corporal e melhora da mobilidade do idoso.

\section{METODOLOGIA}

Este estudo de natureza quantitativa, usando o método quantitativo, foi desenvolvido em duas etapas, sendo primeira uma pesquisa bibliográfica sobre o tema em questão e a segunda uma pesquisa de campo para se penetrar no universo pesquisado. Este tipo de instrumento de pesquisa proporciona captar de forma fácil às informações, possibilitando maior aproximação da realidade e uma maior interação com o sujeito-objeto da pesquisa. (BARDIN,1995)

\subsection{População e amostra}

A população sugerida são 20 idosos, 14 do sexo feminino e 6 do sexo masculino com idades variáveis entre 60 e 65 anos. Foram escolhidos aleatoriamente entre os frequentadores e praticantes de caminhada no Parque Nacional de Teresópolis e que se disponibilizaram a responder o questionário colaborando assim com a pesquisa. Ressalta-se que a quantidade maior de pessoas do sexo feminino se deu pelo fato dessas se mostrarem mais disponíveis a participar da pesquisa. Foi entregue a todos o TCLE e emitido pela Logos University- EUA e após isso foram entregues os questionários. 


\subsection{Procedimentos de coleta}

O desenvolvimento da pesquisa foi através do modelo do (IPAQ) Questionário Internacional de Atividade Física, contendo perguntas objetivas acerca da vida diária, onde ele foi aplicado a 20 idosos com idade superior a 60 anos, e inferior a 65, que faz caminhada no Parque Nacional Serra dos Orgãos em Teresópolis. Antes da coleta de dados no campo, houve um contato prévio com os indivíduos para conhecer suas atividades diárias, para mostrar o objetivo do estudo. O questionário constou de perguntas relativas ao tempo de prática da caminhada, quantos dias na semana, o que levou a praticar a caminhada, como leva a vida diariamente, e seu desempenho na vida diária.

\subsection{Resultados e Discussão}

O Parque Nacional Serra dos Órgãos é um dos cartões postais da cidade de Teresópolis no Rio de Janeiro. Local onde crianças, jovens, adultos e idosos desfrutam de uma bela paisagem e de um ambiente acolhedor, aprazível e em contato direto com a natureza. Totalmente arborizado e pavimentado, o local hoje além de local de lazer e entretenimento, serve também como o point da saúde, onde pessoas de todas as idades, gêneros e classes tem à sua disposição uma vista privilegiada para a prática dos seus exercícios físicos. É nesse panorama que encontramos nosso sujeito-objeto de estudo, o idoso.

\subsection{Da entrevista}

Segundo dados do IBGE possui 6.789 idosos entre homens e mulheres (acima de 60 anos e com menos de 65, a faixa aqui estudada), análogos aos quase 7 milhões nessa mesma faixa etária no restante do território do Brasil conforme recenseamento de 2010. Sendo assim ressaltamos que a nossa pesquisa é indicadora de um pequeno percentual, frente ao número que a cidade apresenta.

Nos gráficos demonstrativos abaixo, é feito o quantitativo da pesquisa e a análise do questionário. A análise dos dados foi obtida através do método de Análise de Conteúdo de Laurence Bardin (1995), que é é uma metodologia de análise de textos que parte de uma perspectiva quantitativa, analisando numericamente a frequiência de ocorrência de determinados termos, construções e referências em um dado texto. Em Comunicação, é freqüentemente usada como contraponto à análise do discurso, eminentemente qualitativa. 
1. Quantas vezes por semana você realiza atividades sentado como lendo, assistindo televisão ou fazendo trabalho com as mãos?

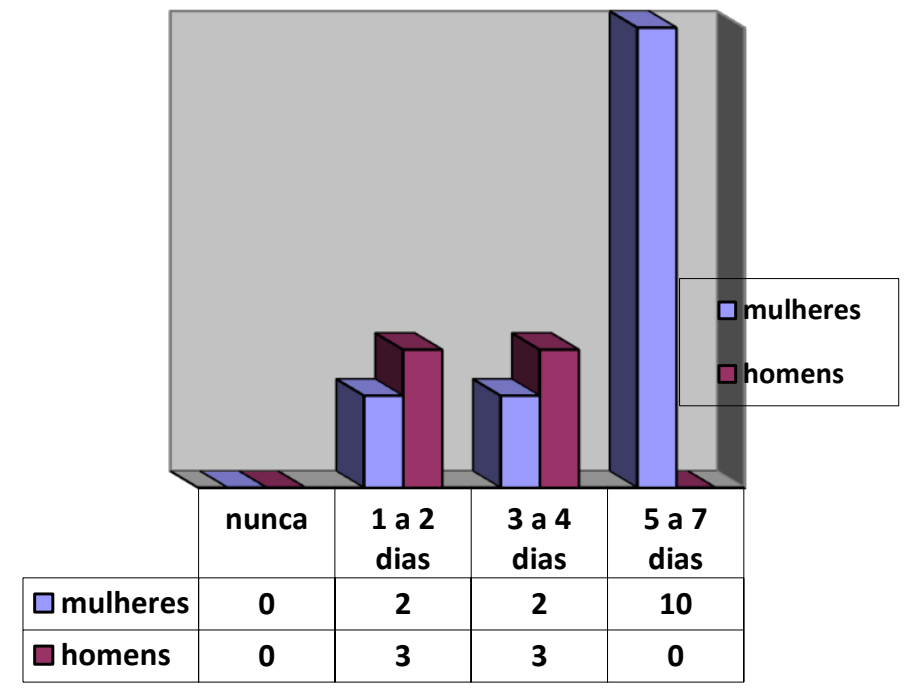

Figura 1

Das 14 mulheres, entre 60 e 66 anos, duas responderam que se exercitam de 1 a 2 dias, e as outras duas de 3 a 4 dias. Os 6 homens entre 60 e 65 anos, três afirmaram que se exercitam de 1 a 2 dias, e as outros três de 3 a 4 dias. O que demonstra um equilíbrio razoável na quantidade de dias, sendo que a quantidade ideal como foi vista anteriormente deve ser prescrita e acompanhada pelo médico.

2. Em média, quantas horas por dia você realiza atividades sentado?

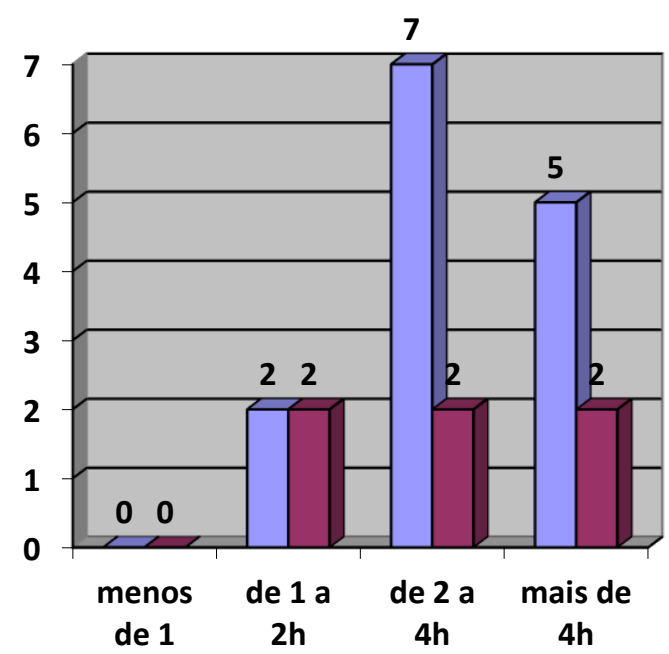

$\square$ mulheres

$\square$ homens

Figura 2 
Nesse item, cinco mulheres e dois homens afirmaram que ficam mais de 4 horas sentadas, as demais 7, responderam que ficam de 2 a 4 horas, o que é uma quantidade de horas razoável, sendo que quem fica mais tempo sentado, deve portanto disponibilizar mais tempo pra se exercitar, e diante da questão anterior, a pessoa que fica mais tempo sentada é quem justamente fica apenas 1 a 2 dias se exercitando. Apenas duas ficam de 1 a duas horas sentadas, Vale ressaltar que a qualidade dos exercícios também importa mais que a quantidade de dias. Dos homens, um apenas fica de uma a duas horas, dois de duas a quatro horas e 3 mais de quatro horas sentados.

\section{Quantas vezes por semana você caminha fora da sua casa ou jardim por qualquer} razão?

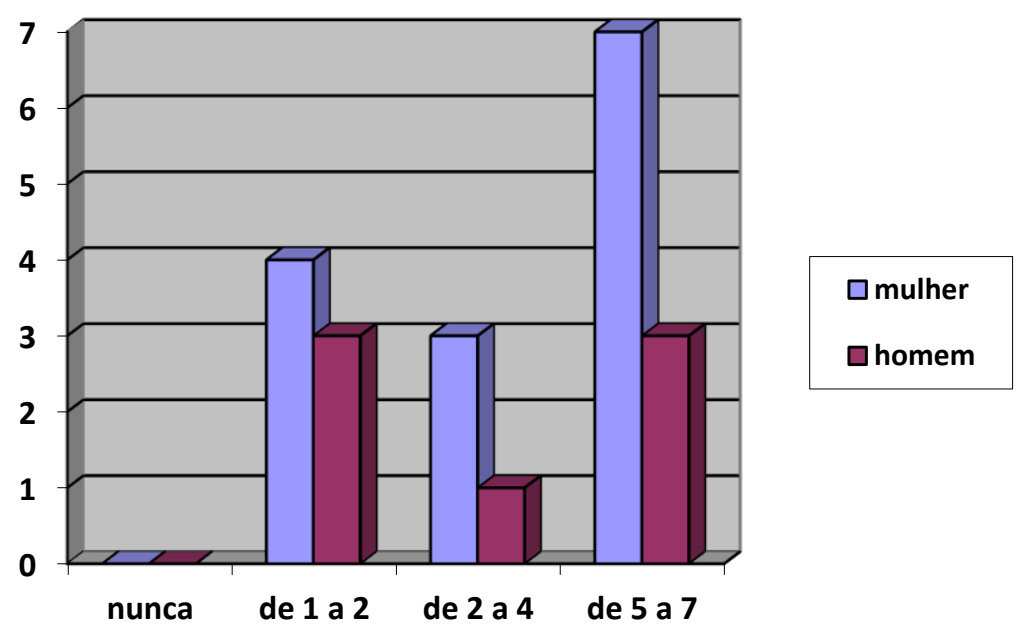

\section{Figura 3}

Em resposta a essa questão, todas as mulheres responderam que de um a dois dias é o tempo que caminham fora de casa, nota-se que elas parecem não ter incluído o tempo que fazem a caminhada habitual, e os homens, 3 deles responderam também que de 1 a 2 dias e os outros 1 apenas de 2 a 4 dias 2 afirmam que de 2 a 4 dias. Os 3 primeiros também parecem não ter incluído o tempo disponibilizado para a caminhada no Parque.

\section{Em média, quantas horas por dia você gasta andando?}




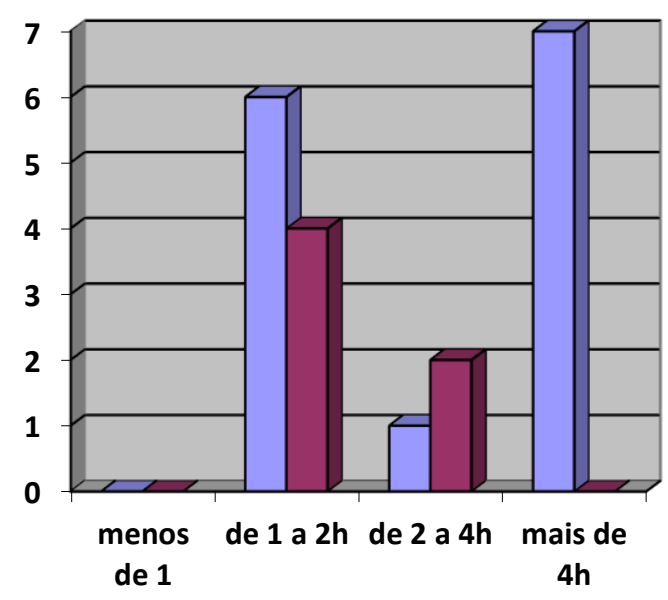

\section{Figura 4}

Nesse item, 6 mulheres responderam que gastam de uma a duas horas por dia andando e apenas uma, justamente a que passa mais de 4 horas sentada, respondeu que gasta de 2 a 4 horas. 7 responderam que gastam mais de $4 \mathrm{~h}$ diárias em movimento. Os homens, 4 responderam que gastam de uma a duas horas e apenas 2 gastam de 2 a 4 horas.

\section{Quantos quarteirões você caminha por semana?}

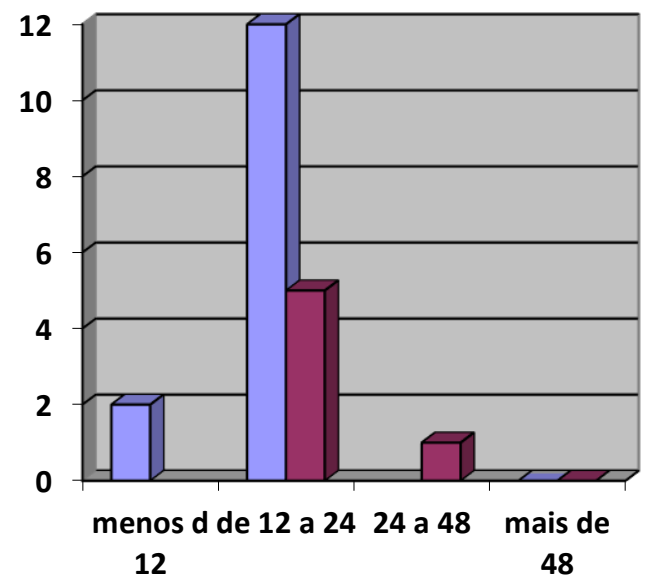

\section{口mulheres \\ 口homens}

\section{Figura 5}

$\mathrm{Na}$ terceira questão, 2 mulheres responderam que caminham menos de 12 quarteirões e 12 responderam que caminham de 12 a 24. Os homens, 5 responderam que caminham de 12 a 24 , enquanto apenas um respondeu que caminha de 24 a 48 , o maior número entre os vinte idosos. 
6.Quantos lances de escada você sobe por semana?

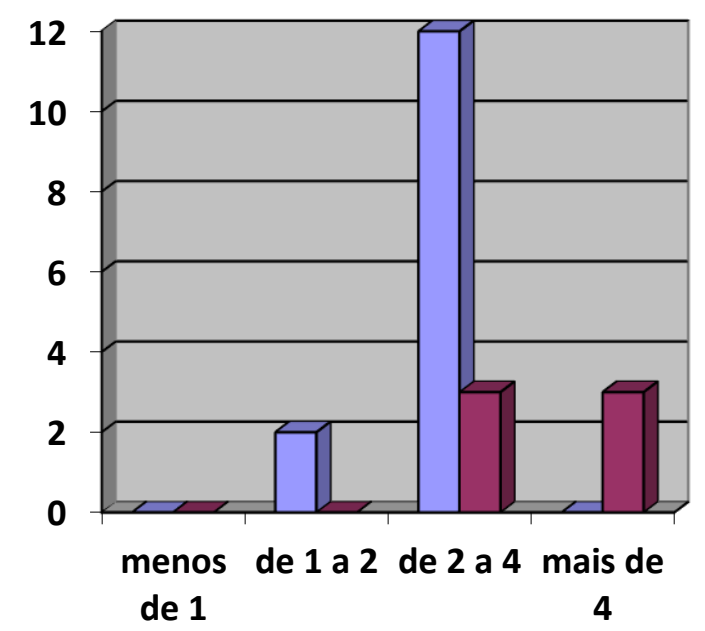

$\square$ mulheres

口homens

Figura 6

Das mulheres, 4 afirmaram que de 1 a 2 lances e as outras doze de 2 a 4 . Quanto aso homens, 3 responderam que de 2 a 4 lances e 3 que sobem de 2 a 4. Essa quantidade é razoável para a faixa etária em questão.

7.Você faz serviços domésticos como lavar pratos ou varrer?

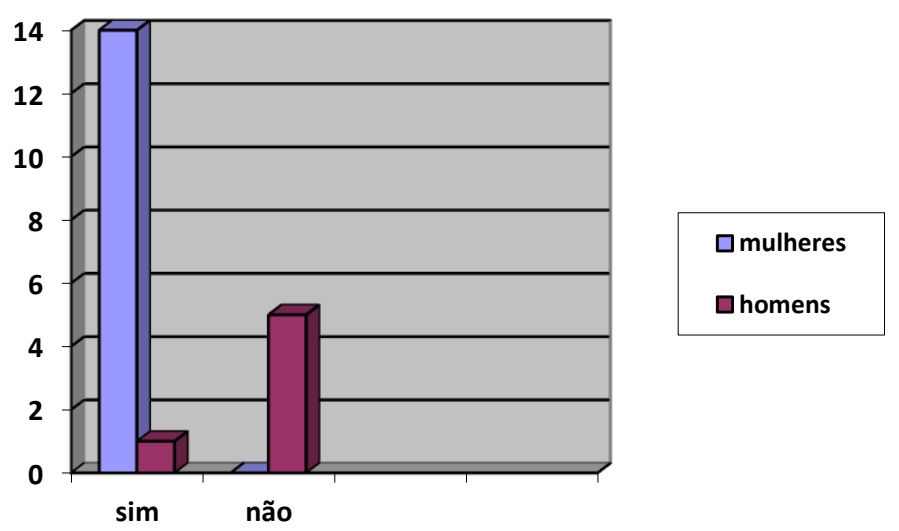

\section{Figura 7}

Todas as senhoras afirmaram que fazem regularmente os serviços domésticos, enquanto apenas 1 dos 6 homens inquiridos afirmaram positivamente fazer serviços domésticos. 
8.Você faz serviços domésticos pesados como aspirar, lavar, esfregar pisos e carregar pesos?

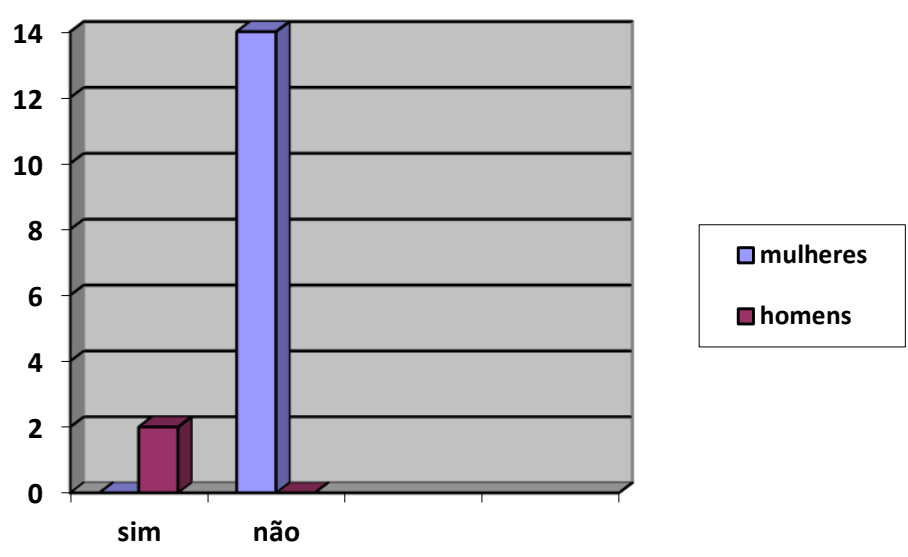

\section{Figura 8}

Das 14 mulheres nenhuma afirmou fazer trabalhos domésticos pesados, enquanto que dos 6 homens, 2 afirmam que sim, fazem, enquanto 4 afirmaram que não executam nenhuma atividade considerada pesada.

\section{Você trabalha ou faz algum trabalho voluntário?}

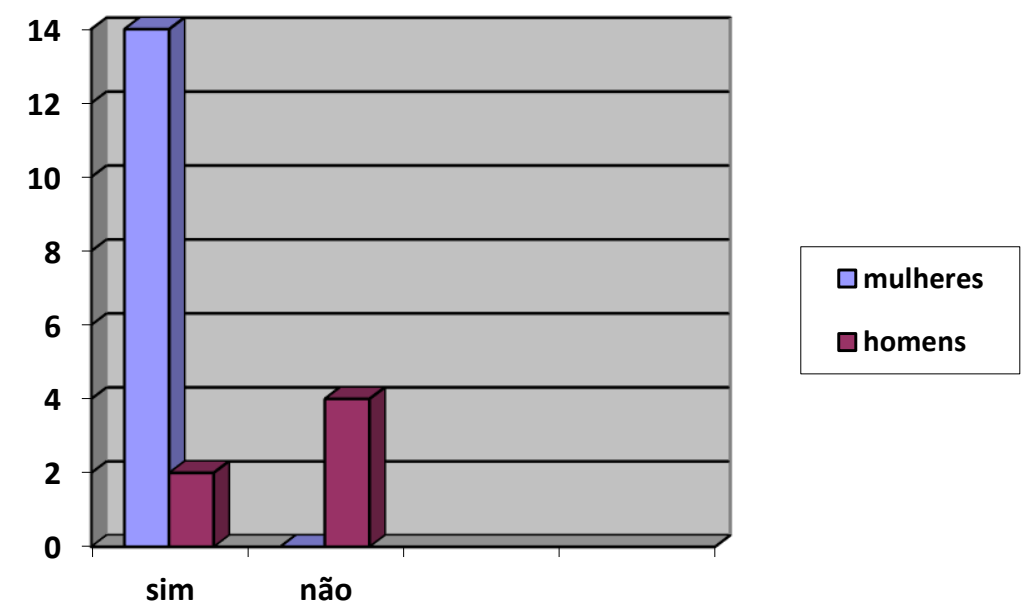

\section{Figura 9}

Nessa questão todas as 14 mulheres afirmam trabalhar de 15 a 30 horas semanais. Uma afirmou que não trabalha, mas parece ter-se confundido por causa da pergunta acerca dos trabalhos voluntários. Dos homens 2 responderam que sim e 4 que não trabalham, porém todos 
afirmam em termos de carga horária que trabalham acima de 20 e menos que 30 horas semanais. Possivelmente também se confundiram com a questão quando se referia a trabalhos voluntários.

10.Qual das seguintes categorias descreve melhor o tipo de atividade requerida no seu trabalho?

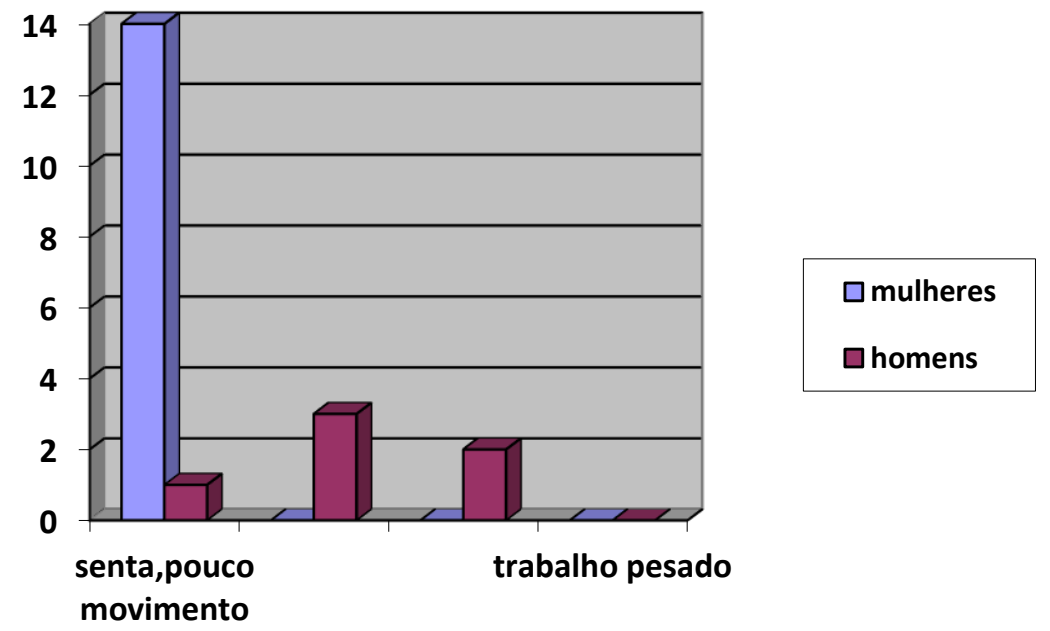

\section{Figura 10}

Nesse ítem todas as 14 mulheres responderam a alternativa 2, que seu trabalho requer que fiquem sentadas ou em pé com alguma caminhada e dos homens, 1 respondeu que sua atividade requer que fique sentado e com pouco movimento dos braços, 2 afirmam que requer que caminhem carregando algum material menor que 25 quilos e 3 afirmam que ficam sentado ou em pé com alguma caminhada.

\subsection{Discussão}

Nota-se aqui que apesar de ter sido um pequeno recorte da realidade, apenas com um grupo de 20 pessoas, tem sido bem maior o número de idosos que caminham na região do Parque Nacional de Teresópolis, o que apesar de interessante, ainda não condiz com a quantidade de idosos em situação de ócio e sedentarismo na cidade. Outro fato a se observar é que as pessoas entrevistadas e as frequentadoras são de classe média a alta conhecida na cidade, embora o lago seja um espaço público alternativo, não se nota a presença em massa de idosos menos abastados economicamente, o que denota um certo elitismo diante da visão do local.

Deduz-se que estes indivíduos se exercitam para ajudar nas suas atividades de vida diária, pois, estudos relatam que a prática de atividade física (caminhada), melhora a capacidade 
funcional, circulatória, contribuindo para o tratamento de várias enfermidades incluindo neuroses de depressão e ansiedade, (ELIOT et al,1992).

As vantagens da prática de exercícios para idosos dependem de como se processa o envelhecimento e da rotina de exercício físico praticada. Sabe-se que os benefícios à saúde ocorrem mesmo quando a prática de atividade física é iniciada em uma fase tardia de vida, por sujeitos sedentários, sendo benéfica inclusive para portadores de doenças crônicas (ELIOT; LONG; BOONE, 1992), prevenindo principalmente as doenças associadas ao sedentarismo, como coronariopatias, diabetes, hipertensão arterial, hipercolesterolemia, acidente vascular cerebral, osteoporose, osteoartrite, e câncer de próstata, mama e cólon intestinal.

Do efeito dos exercícios na prevenção e recuperação das perdas motoras decorrentes do processo de envelhecimento, conhece-se bem a relação entre treinos específicos e a melhora do órgão ou sistema exercitado, como por exemplo, prática de alongamento muscular e ganho de flexibilidade, ou treino de equilíbrio e melhora no desempenho em testes de equilíbrio (JUDGE; UNDERWOOD; GENNOSA, 1993). Para que os efeitos do treinamento de exercícios permaneçam, praticá-los deve tornar-se parte da rotina diária (MARCUS, 1995; MARCUS; RAKOWSKI; ROSSI, 1992; SHEPARD, 1993a; WILLIAMS; LORD, 1995).

De acordo com a análise do questionário aplicado a 20 idosos, sendo 14 mulheres e 6 homens observamos, primeiro a vistas grossas, (pois não questionamos gênero enquanto categoria de análise) numa observação informal no local, é hoje maior o número de mulheres ao de homens que se exercitam.

Todos os entrevistados perceberam uma melhora no desempenho de suas atividades diárias, principalmente no que se refere à força muscular, melhorando a percepção, o desempenho de se locomover sem dificuldade sendo assim independente. O resultado foi positivo, pois através da caminhada regularmente houve uma melhora no sistema fisiológico de um indivíduo idoso e ainda apresentaram perca de um bom percentual gordura.

Além disso, a socialização, o encontro que essas caminhadas proporcionam ajudam à troca de informações, experiências, o contato, que acabam elevando a autoestima e possibilitando ao idoso evitar a solidão que a velhice outrora impunha, diminuindo assim os riscos de depressão, mal que assola toda a humanidade na contemporaneidade e já tida como a doença do século. Além dos dados levantados pela entrevista, a observação e conversa informal foram de grande valia para a conclusão desse trabalho.

Conclui-se assim que é muito bom o nível de atividade física da população amostra, condizente com sua faixa etária e sua expectativa de vida. Mas o que ainda é melhor, mostra a mudança paulatina, gradual, mas contínua do perfil do idoso no país. 


\section{CONSIDERAÇÔES FINAIS}

A atividade física é um ponto importante na qualidade de vida do idoso. No entanto, o tipo de exercício a ser realizado depende do organismo e da vontade de cada um. Segundo especialista, não há nenhuma fórmula predeterminada do que deve ser feito na terceira idade. Por essa razão, dois fatores são preponderantes na escolha da atividade física. Primeiramente, o idoso precisa olhar para si e ver qual a sua capacidade funcional nas atividades do dia-a-dia, como subir as escadas de um ônibus, carregar panelas de pressão, arrumar camas, abaixar-se para ver o forno, por exemplo.

A atividade física irá melhorar sua capacidade de desempenhar essas e outras tarefas cotidianas. Depois procurar a correta avaliação médica, o acompanhamento e somado a esses, a disposição e força de vontade, para fazer dessa etapa da vida, além de útil e prazerosa, também saudável e tranquila, sem os grandes estertores físicos, mentais e emocionais que a velhice costumava carregar. Antigamente a velhice como uma fase de incapacidade física e intelectual que acontece à revelia do ser humano e que impossibilita a sua atenção como cidadão na sociedade. 


\section{REFERENCIAS BIBLIOGRÀFICAS}

ALAN, G. Conhecimento de adultos sobre o papel da atividade física na prevenção e tratamento de diabetes e hipertensão: estudo de base: Rio de Janeiro, RJ, v.25, n.3, mar./2009.

BARBOSA, MT. Como avaliar quedas em idosos. Rev Ass Med Brasil. 2001;

BRASIL Gabinete do Ministro de Estado da Saúde (BR). Portaria No 1.395 de 9 de dezembro de 1999: aprova a Política Nacional de Saúde do Idoso e dá outras providências. Diário Oficial [da] República Federativa do Brasil, 13 Dez 1999. Seção I, n.237-E, p.20-4.

BRITO, Lucia; FRANCIELLO, Maria. Construção de instrumento para avaliação sócio funcional em idosos: Texto e contexto- enferm. Florianópolis, SC, v.1, n.2, abr./jun.2008.

CASPERSEN CARL J. Ph.D. M.P.H. Exercise and Sport Sciences Reviews: January 1989 Volume 17 - Issue 1 - p 423-474.

ELIOT, R. S.; LONG, D. R.; BOONE, J. L. Rehabilitation. In: ELIOT, R.S. (Ed.). Stress and the heart. New York: Futura, 1992.

GUIMARÃES LHCT, Comparação da propensão de quedas entre idosos que praticam atividade física e os idosos sedentários.

IBGE. População, pirâmide etária. $\quad$ Disponível em https://cidades.ibge.gov.br/brasil/rj/teresopolis/panorama Acesso em março de 2012.

JUDGE JO, UNDERWOOD M, GENNOSA T. Exercise to improve gait velocity in older persons. Arch Phys Med Rehabil. 1993;74:400-6.

MARCUS, B. H.; RAKOWSKI, W.; ROSSI, J.S. Assessing motivational readiness and decision making for exercise. Health Psychology, USA, v. 11, n. 4, p. 257261, 1992.

MATSUDO SMM. Envelhecimento \& atividade física. Londrina: Midiograf; 2001. 
Avaliação do Idoso - Física \& Funcional.2ª .ed. São Paulo.Ed. Phorte,2003.

MEINEL, K; SCHNABEL, G. Motricidade II: O desenvolvimento motor do ser humano. Rio de Janeiro: [s.n.], 1984.

OKUMA, Silene Sumire.O idoso e a atividade física: Fundamentos e pesquisa. Campinas São Paulo. 5. ed. Papirus, 2009.

SPIRDUSO WW. Dimensões físicas do envelhecimento. $2^{\mathrm{a}}$ ed. São Paulo: Manole; 2005.

SOARES, E. et al. Eficácia de programas de educação para adultos portadores de hipertensão arterial: Brasília, DF, v.59, n.2, jul./agos.2006.

PAFFENBARGER RS, HYDE RT, WING AL, Hsieh CC. Physical activity, all-cause mortality, and longevity of college alumni. N Engl J Med. 1986; 6:314:605-13.

PERRACINI MR, RAMOS, LR. Fatores associados a quedas em uma coorte de idosos residentes na comunidade. Rev Saúde Pública. 2002;

POZENA, Regina. CUNHA, Nanci. Projeto "Construindo um futuro saudável através da prática da atividade física diária". Rev. Dep. Psicol.,UFF v.18 n.2 Niterói jul./dez. 2006.

RIKLI RE, Jones CJ. The reliability and validity of a six-minute walking test as ameasure of physical endurance in older adults. J Aging Phys Act. 1998;6:363-75.

SHOUERI JR, R. et.al. Crescimento populacional: aspectos demográficos e sociais. [s.1]: E.T.\& Papaleo, 1994.

SILVA, A C. et. Al. Problemas do idoso, um desafio social. São Paulo: Editora da USP, 1984 WEINBERG, R., \& GOULD, D. Fundamentos da Psicologia aplicada ao exercício e ao esporte. ARTMED, Porto Alegre: ARTMED.2001. 